\title{
Enhancement of Quality of Service Parameters in Wimax Mobile Networks
}

\author{
${ }^{1}$ Chandra and ${ }^{2}$ HelenPrabha \\ ${ }^{1}$ Faculty of ECE, St. Peter's University, Chennai 600054, Tamil Nadu, India \\ ${ }^{2}$ Faculty of ECE, R.M.D. Engineering College, Anna University, Chennai 601208, Tamil Nadu, India
}

Received 2012-07-11, Revised 2012-09-14; Accepted 2012-11-07

\begin{abstract}
Next generation mobile networks are expected to provide seamless personal mobile communication and QoS. Since the demands for high speed Internet access as well as multimedia service had increased for last mile broadband access, the Scheduling is one of the most important components of BWA systems that affect system QoS provided to users. Priority queuing gives better throughput than the existing scheme. The IEEE 802.16 standard is a rapidly developing technology for broadband wireless access system. It supports QoS and has very high transmission rate. WiMAX is a standard wireless technology which is used to improve internet access and the multimedia services at very high speed to the end user. The purpose of QoS is to provide a definitive connection to the users who are intrested to connect to the WiMAX tower. In this study we propose a scheme to improve the QoS in the WiMAX system by increasing the throughput and decreasing the jitter. This study is based on the use of two scheduling algorithms to calculate the data in the MAC layer in order to provide maximum throughput. Moreover we propose a scheme to calculate the jitter between the various mobile users. The scheme performance is investigated through simulation. Priority Queuing gives better throughput compared to the Random Early Detection. Also jitter is minimized in priority queuing.
\end{abstract}

Keywords: QoS, WiMAX, MAC Layer, IEEE 802.16, Packet Scheduling

\section{INTRODUCTION}

Demands for high speed Internet access and multimedia service for residential and business customers had increased for last mile broadband access. The IEEE 802.16 standards (or WiMAX) are an emerging broadband wireless access technology to provide users with high speed multimedia services. WiMAX is a part of fourth Generation (4G) wireless communication technology. WiMAX supports point-to point or point -to-multipoint connection. Multiple standard of WiMAX such as 802.16e, 802.16b for mobile connectivity from fixed location. The IEEE 802.16 supports QoS and has very high transmission rate. The key part of 802.16-packet scheduling, was undefined and is an open issue. This issue has been considered in our work and a scheduling algorithm is developed to improve the efficiency of the WiMAX system.

\subsection{Characteristics of WiMAX}

WiMAX is a worldwide Interoperability for microwave access. It is a promising communication technology for wirelessly delivering high-speed Internet service to large geographical areas. WiMAX far surpasses the $30 \mathrm{~m}$ wireless range of a conventional WiFi Local Area Network (LAN), offering a metropolitan area network with a signal radius of about $50 \mathrm{Km}$. WiMAX as a standards-based technology enabling the delivery of last mile wireless.

WiMAX operates on both licensed and non-licensed frequencies, providing a regulated environment and viable economic model for wireless carriers. The basic difference between WiMAX and Wi-Fi are cost, speed, distance and so on. WiMAX coverage is about 30 miles and Wi-Fi coverage is very limited to some small indoor area. While Wi-Fi offer quality of services to fixed

Corresponding Author: Chandra, Faculty of ECE, St. Peter's University, Chennai 600054, Tamil Nadu, India 
Ethernet where packets are precedence on their tag. Hotspots of Wi-Fi are usually backhauled over ADSL in small business, cafe. Therefore to get access is normally highly challenging. The uploading speed of Wi-Fi as compared to WiMAX is also very low rate among internet and router. OFDM is popular for wideband communications today by way of low cost digital signal processing components.

\subsection{Media Access Control Layer}

The WiMAX MAC uses a scheduling algorithm for which the subscriber station needs to complete only once for initial entry into the network. After a network entry is allowed the subscriber station is allocated an access slot by the base station. The time slot can enlarge and contract, but remains assigned to the subscriber station, which means that other subscribers cannot use it. In addition to being stable under overload also be more bandwidth efficient. The scheduling algorithm also allows the base station to control Quality of service (QoS) parameters by balancing the time-slot assignments among the application needs of the subscriber station. The key part of 802.16- packet scheduling, was undefined and is an open issue (Jiang and Tsai, 2006). This issue has been considered in our work and a scheduling algorithm is developed to improve the efficiency of the WiMAX system.

\subsection{Quality of Service}

QoS refers to the resource reservation control mechanism. QoS is the ability to provide different priority to different applications, users, or data flows, or to guarantee a certain level of performance to a data flow. For example a required bit rate, delay, jitter, packet dropping probability and/or bit error rate may be guaranteed. Quality of service guarantees are important if the network capacity is insufficient, especially for realtime streaming multimedia applications. They often require fixed bit rate and are delay sensitive. Whenever a packet is transmitted from the source to the destination, the following problems occur.

\subsection{Low Throughput}

Due to varying load of other users sharing the same network resources, the bit rate (the maximum throughput) that can be provided to a certain data stream may be too low for real-time multimedia services if all data streams get the same scheduling priority.

\subsection{Dropped Packets}

The routers might fail to deliver (drop) some packets if their data is corrupted or they arrive when their buffers are already full. The receiving application may ask for this information to be retransmitted, possibly causing severe delays in the overall transmission.

\subsection{Jitter}

Packets from the source will reach the destination with different delays. A packet's delay varies with its position in the queues of the routers along the path between source and destination. This position can vary unpredictably. This variation in delay is known as jitter and can seriously affect the quality of streaming audio and/or video.

\subsection{Related Work}

The QoS supported by the IEEE 802.16 standard operates within a full set of parameters that permit service differentiation for the service connections (Lai et al., 2011). Broadband Wireless Access (BWA) has become the best way to meet escalating business demand for rapid Internet connection and integrated data, voice and video services. Scheduling plays an important role in providing Quality of Service (QoS) support for multimedia networks. A cross layer scheduler provides (Liu et al., 2005) diverse QoS guarantees. With WiMAX the issue of interference is lessened which is proposed in our scheme. WiMAX is a standards initiative, its purpose is to ensure that the broadband wireless radios manufactured for customer use interoperate from vendor to vendor. IEEE standard 802.16 BWA systems offer true differentiated broadband services at minimal cost (Jin and Min, 2007). Jiang and Tsai (2006) the author presents a design process for Wireless Metropolitan Area Network (WMAN), which supports QoS but packet scheduling was undefined and is an open issue. The strategy proposed in (Taddia and Mazzini, 2006) is the impact of the insertion of routers implementing a nonpre-emptive priority queue mechanism in a chain of simple First-In-First-Out (FIFO) routers on the end-toend jitter experienced by high and low priority packets. Chowdhury et al. (2012) QoS parameters like system capacity utilization are considered. The communication between multiple medium access control technologies and the network layer is enabled in (Barbu and Fratu, 2011). Unfortunately previous schemes do not support both scheduling and throughput in an efficient manner. There is no clear definition or implementation detail about the algorithm.

In this study we propose a detail description about WiMAX. For Our proposed scheduling algorithms, priority queuing and Random Early Detection the scheme performance is investigated through simulation. 


\section{MATERIALS AND METHODS}

\subsection{Overview/Problem Identification}

The IEEE 802.16 system defines five types of service: Ungranted Service (UGS), the real time Polling Service (rtPS), extended real-time Polling Service (ertPS), non real time Polling Service (nrtPS) and Best Effort (BE).The existing algorithm controls the bandwidth allocated to them. These service connections can be created, changed or deleted by issuing Dynamic Service Addition (DSA), Dynamic Service Change (DSC) and Dynamic Service Deletion (DSD) messages. The Down Link (DL) from the Base station to the user operates on a Point-to-Multipoint basis. In EBA (Lai et al., 2011) an efficient QoS control protocol design in Upper Link (UL) connections of the IEEE 802.16 system is proposed, where the study was concentrated on individual Subscriber station's location as a fixed one. Since each connection is provided with different service oppurtunities the time taken to process the frame to identify the service type and send them through the various connections decreases the efficiency of the system. The method adopted for ertPS (Kim et al., 2011) supports both multicast and broadcast polling services under collision. The Best Effort Service (BE) is starved in the UBA whereas on the existing system the bandwidth allocated to it varies depending on the spare bandwidth from the other two service categories.

\subsection{Existing Algorithm Discription}

Earliest Deadline First is applied to rtps, which requires more overhead to be implemented. We have to keep track of the absolute deadline in a long data structure, which could be a tedious work. The system may become complex for this reason and the processing of the packets are slowed down while searching for the deadline value.

Weighted Fair Queuing is applied to nrtps, which requires multiple weights for the data, which might increase the overheads of the packets. Increasing the overheads of the data will lead to complex processing of data and introduce delay in the transfer of a packet. WFQ also requires individual FIFO queue for all its weight. This increases the complexity of the hardware and the cost of the system to a greater value.

\subsection{Proposed Algorithm Discription Priority Queing}

Basically our analysis investigates Quality of service in a wireless technology. QoS in Broadband Wireless
Access (BWA) is a difficult and complicated business (Alavi et al., 2005), as it adds an unpredictable radio link and potentially heavy user contention to the usual nondeterministic behaviour of IP packet networks. Carriers therefore need to be aware of QoS. One way of providing QoS is to design a scheduling algorithm such that it decreases the delay, packet loss and increase the throughput of the system. Queuing theory has been used often in modelling multiple access scheme or transmission delay in communication system (Chen et al., 2011).

Figure 1 Shows a modelling of priority queue which explains the flow of data in the scheduler when it enters the MAC layer of the base station. Priority queing is a scheduling algorithm based on the priority of the data. This queing strategy allows traffic to be classified as high, normal, medium or low priority. If there is any highpriority traffic, it is transmitted first, then medium-priority traffic and so on. The low priority traffic may get delayed in the network until the higher priority data are processed.

Whenever a packet arrives at the input of the base station, the packets are checked for priority. If the packets are the high priority type then it is queued in the separate queue, if the packets are Medium (Med) priority then it is enqueued in the separate queue and so on. Analytical modelling and performance evaluation of priority queing systems have received significant research efforts in the telecommunication community (Jin and Min, 2007).

\subsection{Random Early Detection}

Random Early Detection (RED) is a queue management algorithm. Figure 2 shows the modelling of RED queue algorithm It is also a congestion avoidance algorithm. RED monitors the average queue size and drops packets based on statistical probabilities. If the buffer is almost empty, all incoming packets are accepted. As the queue grows, the probability of dropping an incoming packet grows too.

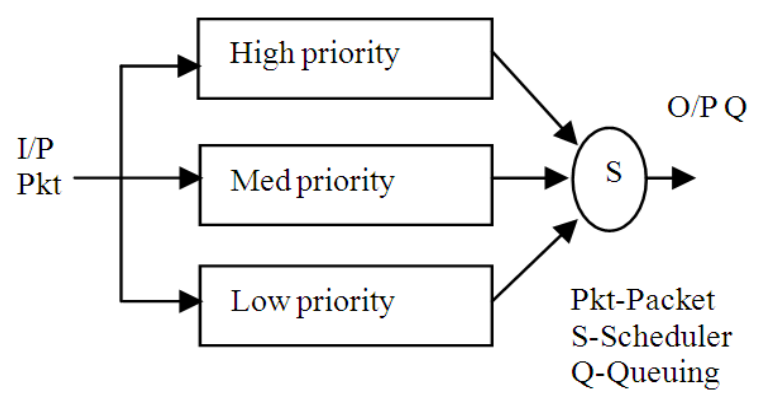

Fig. 1. The Priority queue algorithm model 


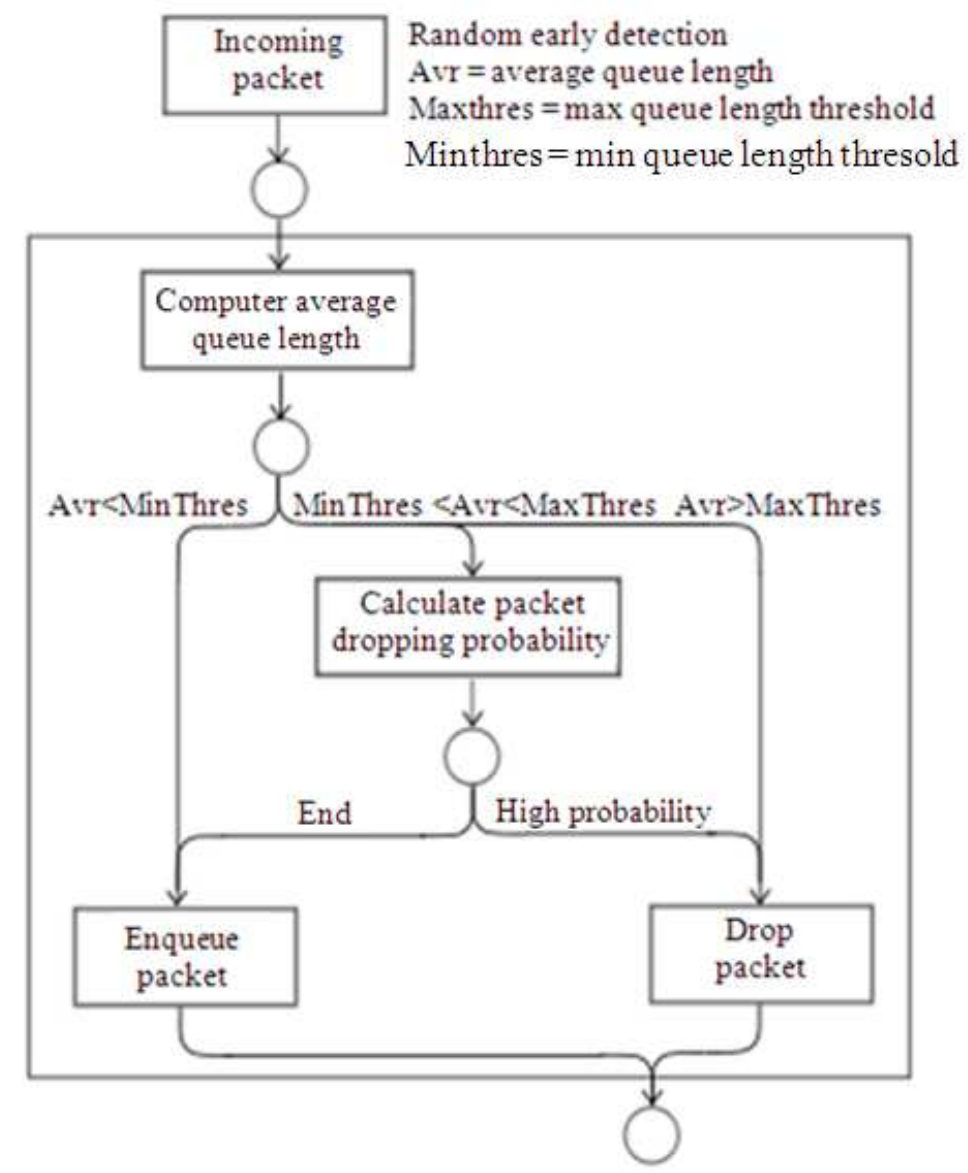

Fig. 2. The RED queue algorithm model

When the buffer is full, the probability has reached 1 and all incoming packets are dropped. RED is more fair than tail drop, in the sense that it does not possess a bias against burst traffic that uses only a small portion of the bandwidth. The more a host transmits, the more likely it is that its packets dropped as the probability of a host's packet being dropped is proportional to the amount of data it has in a queue. Early Detection helps avoid global synchronization.

RED takes a proactive approach to congestion. Instead of waiting until the queue is completely filled up, RED starts dropping packets with a non-zero drop probability after the average queue size exceeds a certain minimum threshold. A drop probability ensures that RED randomly drops packets from only a few flows, avoiding global synchronization. A packet drop is meant to signal the TCP source to slow down. Responsive TCP flows slow down after packet loss by going into slow start mode. This ensures that the congestion is avoided in the network and the consistency of the connection remains the same.

\subsection{Advantages of the Proposed Scheme}

The priority queue has the advantage of attending the high priority traffic in the network rather than a low priority. The priority queue is implemented with a Drop Tail facility to manage the queue. This allows the queue to fill up to the maximum size with the desired packets and when the queue overflows the tail or the incoming packets are dropped.

The response time has been small in priority queuing, which takes only a few bits in the whole header. The overhead created by the priority queuing in the TCP packets are also very less which makes the system to be easy for implementation. 
Random Early Detection (RED) provides both congestion recovery and congestion avoidance. It fixes a lower and an upper threshold in the queue and calculates the drop probability of the incoming packets accordingly. The packets are dropped from the queue whenever the drop probability of a certain packet is high and the mean length of the queue is more than the upper threshold. Thus the RED algorithm helps in congestion avoidance. RED acts as a congestion avoidance in packet switched network (Floyd and Jacobson, 1993).

\section{RESULTS}

\subsection{Simulation Setup}

In order to evaluate and analyze the performance of our scheme, we have developed a simulation tool in NS2 based Linux-Fedora. The goal of the experiment is to show that the proposed scheme can provide QoS support in terms of throughput and jitter.

As depicted in Fig. 3 the simulation starts with one base station and four mobile users in their original positions. As the simulation proceeds, different users tend to move around the scenario. This has ensured that the users possess mobility in the environment. Users constantly connect to the base station to transfer data between them. In this scenario shown, the users move around the coverage area of the base station to become a mobile user and they keep transmitting data with the base station. This is shown by the bursts of signals when the users are interested in sending a packet to the base station. At the start of the simulation the users obtain a wireless channel to transmit the data and remember their slot and frequency to send it.

Figure 4 shows the throughput of the system with the implementation of the Priority Queue algorithm which is first implemented. A priority queue is a collection of elements that each element has been assigned a priority and such that the order in which elements are deleted and processed comes from the following rules:

- An element of higher priority is processed before any element of lower priority

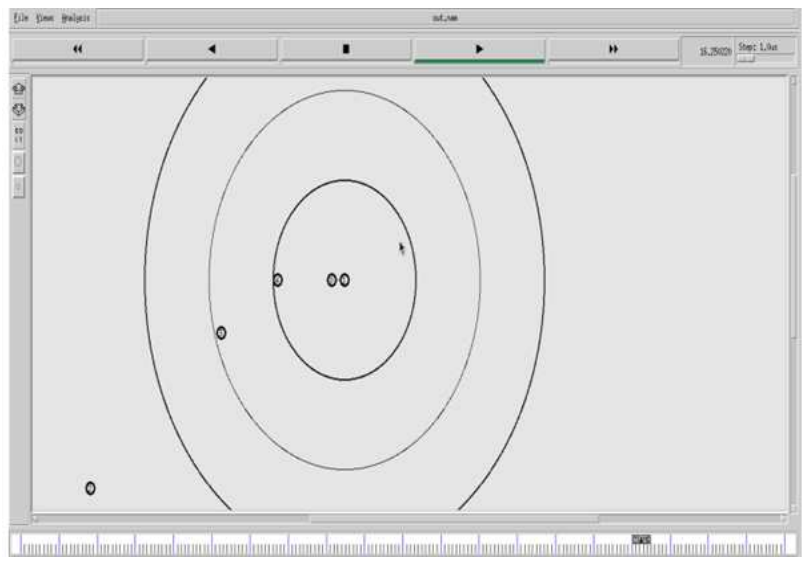

Fig. 3. The Simulated model of four nodes position

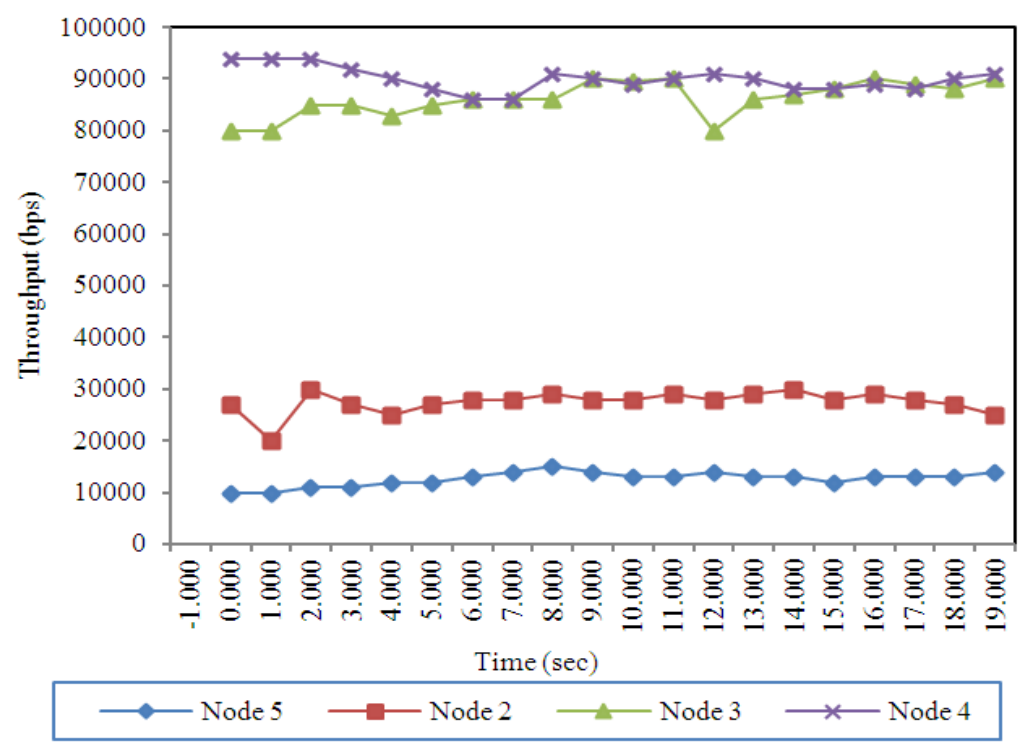

Fig. 4. The Throughput graph of priority queue 


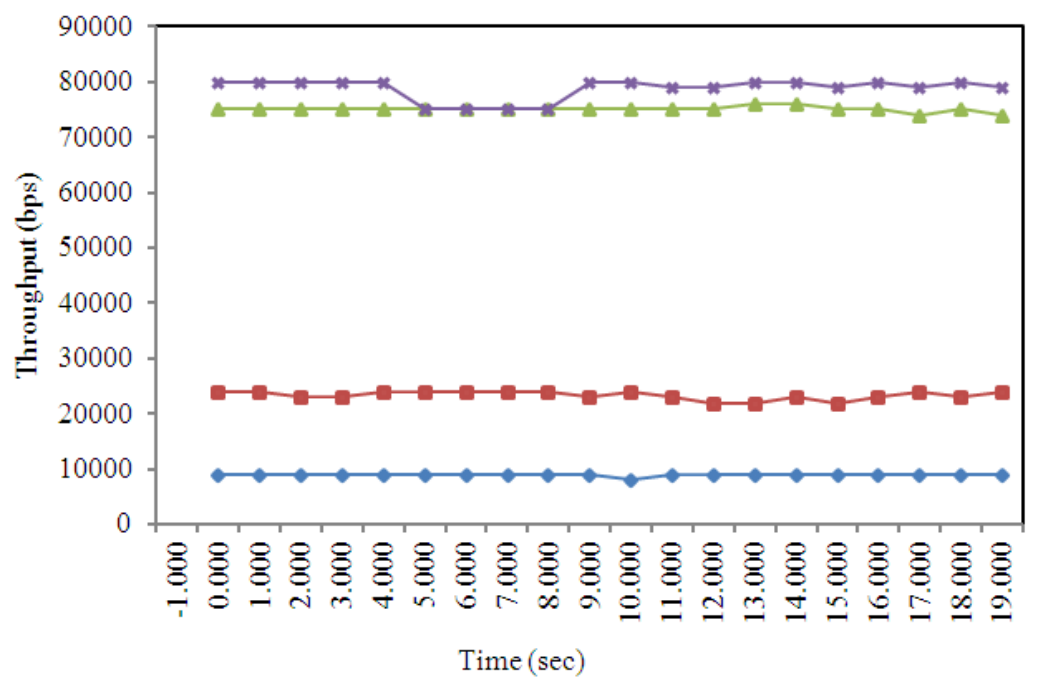

$\longrightarrow$ Node $5 \longrightarrow$ Node $2 \longrightarrow$ Node $3 \longrightarrow$ Node 4

Fig. 5. The Throughput graph of RED queue

- Two elements with the same priority are processed according to the order in which they added to the queue

The result shows those variations in the throughput of the users according to the priority in their data. Throughput also varies due to the distance between them. This is because of the bandwidth allocated to the users and the distance between them from the base station. The variation shows that more often the users demand a high priority data or send a high priority data.

Jitter is another parameter for consideration in the QoS of the WiMAX system. Jitter is defined as the variations in the Delay of the user data.

In Table 1 the various values experienced by the users in the simulation scenario are shown. The jitter has also risen in the simulation when the network has got congested with lots of data. Jitter value for certain nodes has seen increasing to a value of over $1000 \mathrm{~ms}$ for node 2 and has been very low in the order of nanoseconds for node 3 and node 5 .

Figure 5 Shows the throughput graph for the Random Early Detection Queue. RED also a congestion avoidance algorithm. The graph indicates that the throughput of the system remains in a steady state unless congestion is sensed in the network. Then the packets are dropped from the queue depending on the probability of the packets being dropped.
Table 1. Jitter in the priority system

\begin{tabular}{lllll}
\hline $\begin{array}{l}\text { Time } \\
(\mathrm{sec})\end{array}$ & $\begin{array}{l}\text { Jitter2 } \\
(\mathrm{ms})\end{array}$ & $\begin{array}{l}\text { Jitter3 } \\
(\mathrm{ms})\end{array}$ & $\begin{array}{l}\text { Jitter4 } \\
(\mathrm{ms})\end{array}$ & $\begin{array}{l}\text { Jitter5 } \\
(\mathrm{ms})\end{array}$ \\
\hline 0.0000 & 0 & 0 & 0 & 0 \\
1.0000 & 0 & 0 & 0 & 0 \\
2.0000 & 0 & 0 & 0 & 0 \\
3.0000 & 0 & 0 & 0 & 0 \\
4.0000 & 0 & 0 & 0 & 0 \\
5.0000 & 0 & 0 & 0 & 0 \\
6.0000 & 0 & 0 & 0 & 0 \\
7.0000 & 0 & 0 & 0 & 0 \\
8.0000 & 0 & 0 & 0 & 0 \\
9.0000 & 0 & 0 & 0 & 0 \\
10.0000 & 0 & 0 & 0 & 0 \\
11.0000 & 0 & 0 & 0 & 0 \\
12.0000 & 0 & 0 & 0 & 0 \\
13.0000 & 0 & 0 & 0 & 0 \\
14.0169 & 0 & 0 & 0 & 0 \\
15.0089 & 1000 & 8 & 62.5 & 0.5 \\
16.0089 & 1000 & $1.77636 \mathrm{e}-12$ & 62.5 & $1.11022 \mathrm{e}-13$ \\
17.0089 & 1000 & $1.77636 \mathrm{e}-12$ & 62.5 & $1.11022 \mathrm{e}-13$ \\
18.0089 & 1000 & 0 & 62.5 & 0 \\
\hline
\end{tabular}

In Table 2 the various Jitter values for the RED Queue are shown. It shows a large variation in the jitter value due to the congestion that is taking place in the network. Jitter value as high as $4008 \mathrm{~ms}$ are noted for node 2 and very low value of $6.25 \mathrm{~ms}$ is noted for node 3 and node for the delay occurred. 
Chandra and HelenPrabha / American Journal of Applied Sciences, 9 (12) (2012) 1906-1915

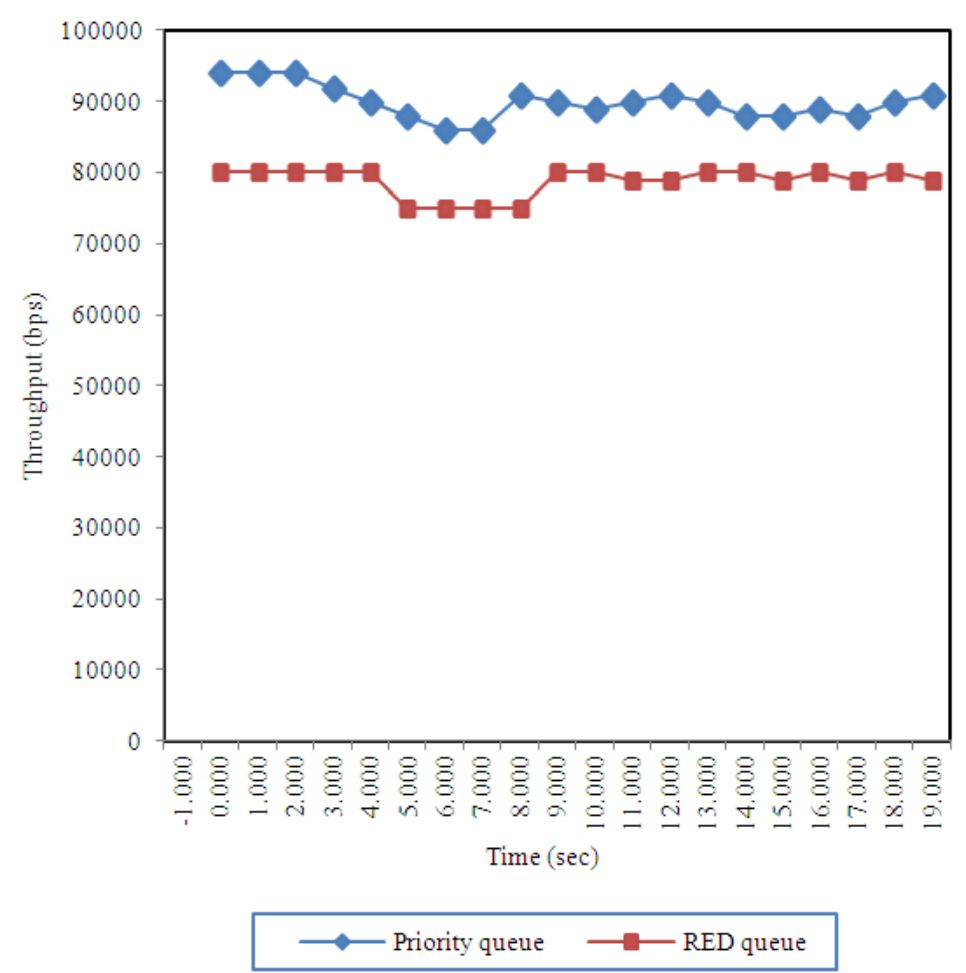

Fig. 6. Comparing node 4 throughput

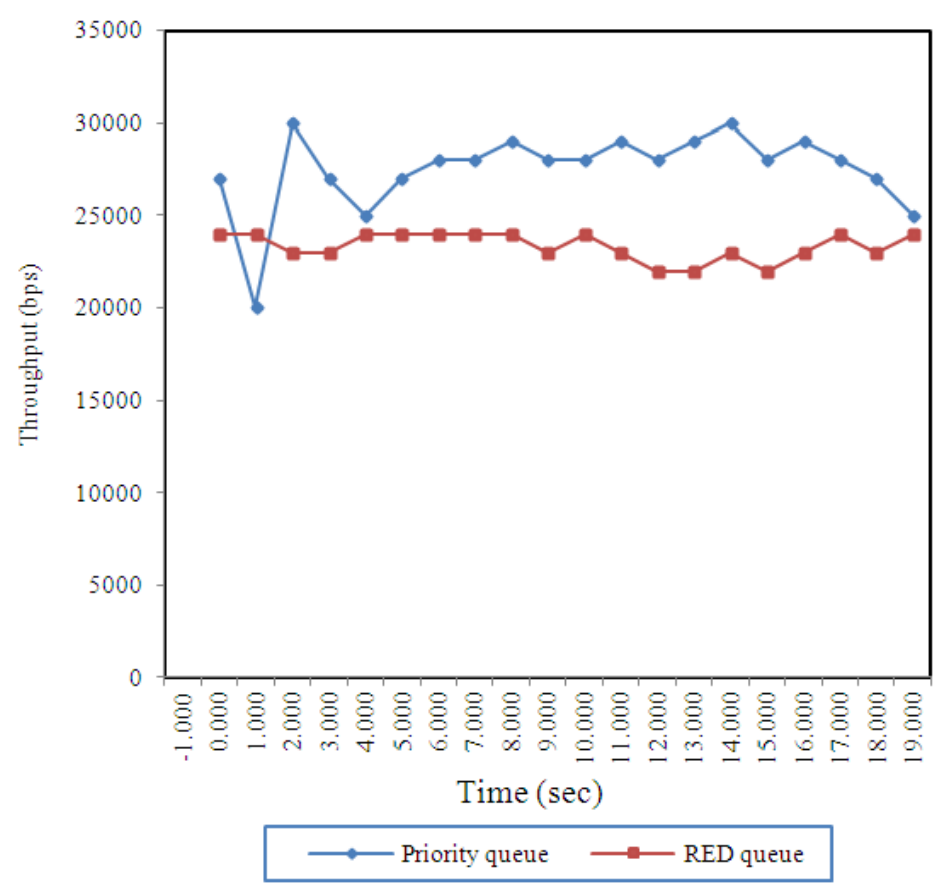

Fig. 7. Comparing node 2 throughput 


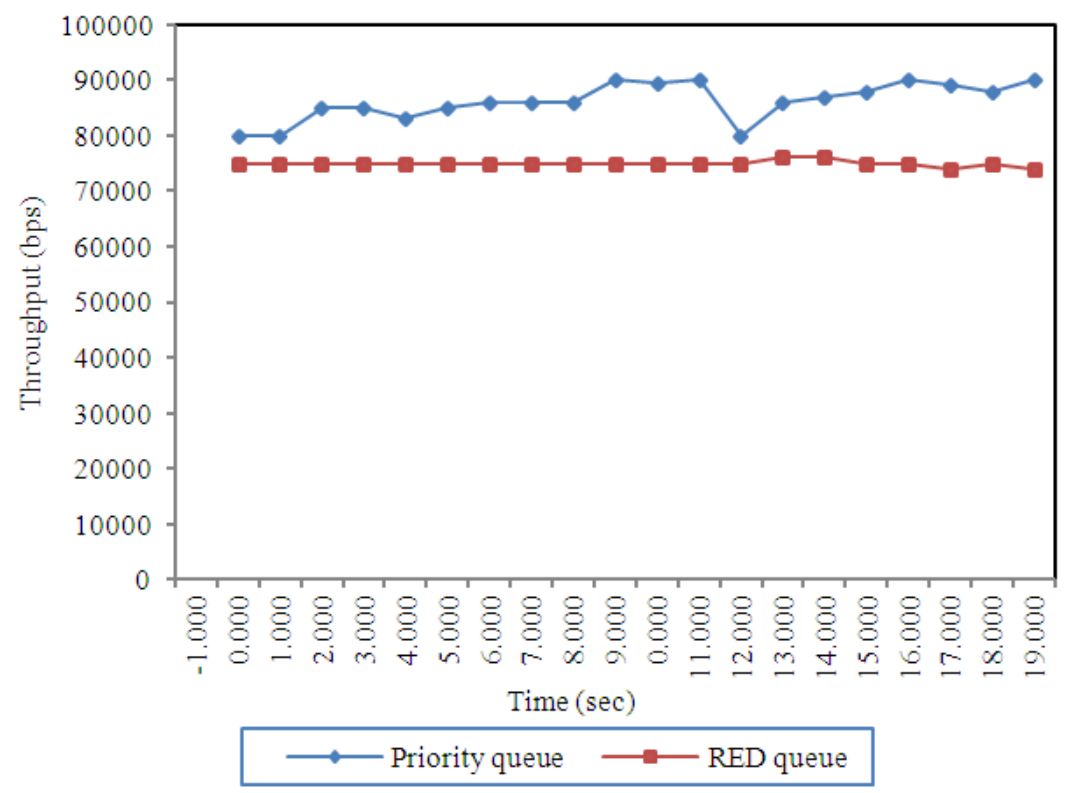

Fig. 8. Comparing node 3 throughput

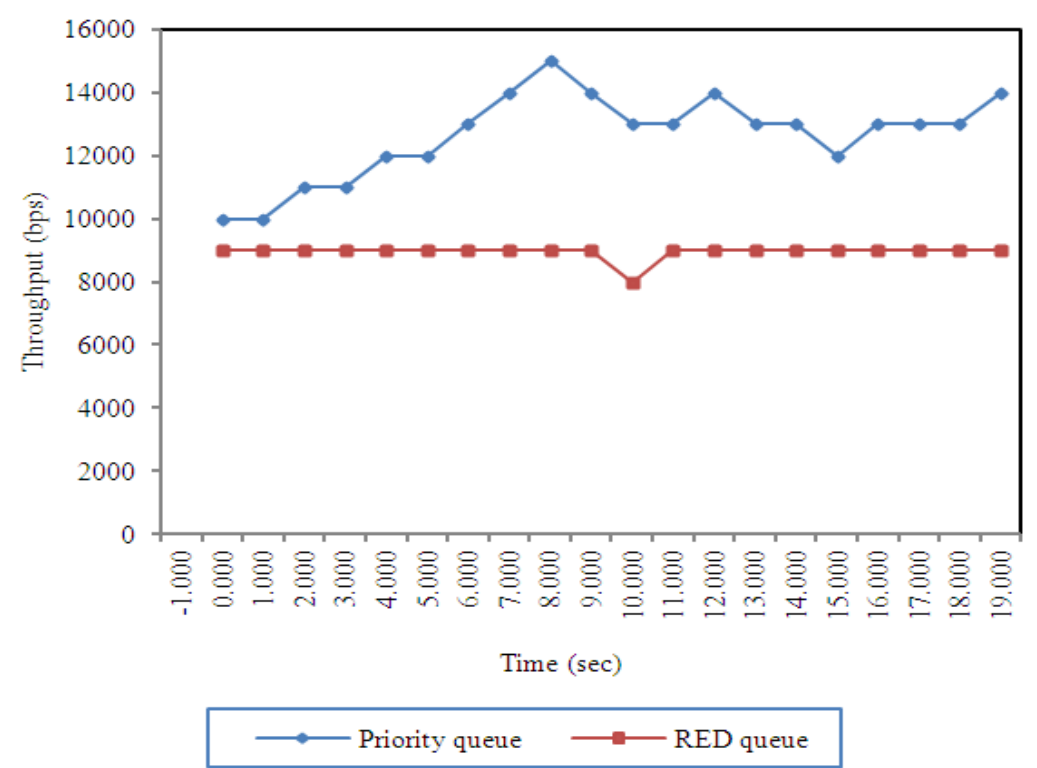

Fig. 9. Comparing node 5 throughput

In Fig. 6 shows a comparison of the throughput for the two algorithms being used. The priority queue promises to give higher throughput in the system.

In Fig. 7 shows the comparison of the throughput for the node 2 of the system. Here the throughput of the priority queue varies abruptly where as in the RED queue the throughput remains as constant as possible thereby avoiding congestion in the system.

Figure 8 compares the throughput of the node 3.The throughput here is maximum for priority queue, however the throughput of the RED queue is constant over a certain period thereby establishing the stability of the system. 
Table 2. Jitter in the red system

\begin{tabular}{lcccr}
$\begin{array}{l}\text { Time } \\
(\mathrm{sec})\end{array}$ & $\begin{array}{c}\text { Jitter2 } \\
(\mathrm{ms})\end{array}$ & \multicolumn{1}{c}{$\begin{array}{c}\text { Jitter3 } \\
(\mathrm{ms})\end{array}$} & \multicolumn{1}{c}{$\begin{array}{c}\text { Jitter4 } \\
(\mathrm{ms})\end{array}$} & \multicolumn{1}{c}{$\begin{array}{c}\text { Jitter5 } \\
(\mathrm{ms})\end{array}$} \\
\hline 0.278956 & 0.000 & 0.000 & 0.00000 & 0.00000 \\
1.01618 & 1016.180 & 459.443 & 63.51130 & 28.71520 \\
2.70818 & 100.000 & 0.000 & 6.25000 & 0.00000 \\
3.00118 & 3008.180 & 100.000 & 188.01100 & 6.25000 \\
4.00119 & 4008.180 & 100.000 & 250.51100 & 6.25000 \\
5.20818 & 100.000 & 6.996 & 6.25000 & 0.43725 \\
6.27896 & 6208.18 & 29.225 & 388.011 & 1.82656 \\
7.001197008 .18 & 100.000 & 438.01100 & 6.25000 \\
8.20818 & 1006.996 & 6.25000 & 0.43725 & \\
9.20818100 & 6.996 & 6.25000 & 0.43725 & \\
10.279000277 .771 & 0.00000 & 17.3607 & \\
11.279 & 000444.458 & 0.00000 & 27.7786 & \\
12.279 & 000444.458 & 0.00000 & 27.7786 & \\
13.279 & 000444.458 & 0.00000 & 27.7786 & \\
14.279 & 000444.458 & 0.00000 & 27.7786 & \\
15.279 & 000444.458 & 0.00000 & 27.7786 & \\
16.0012 & 000444.458 & 0.00000 & 27.7786 & \\
17.279000444 .458 & 0.00000 & 27.7786 & \\
18.279 & 000444.458 & 0.00000 & 27.7786 & \\
19.279 & 0.00 & 444.458 & 0.00000 & 27.7786 \\
\hline
\end{tabular}

Table 3. Comparing QoS parameters

\begin{tabular}{llll}
\hline Subscriber & Throughput (bps) & & \\
\hline station & priority queueing & Red queue & EBA \\
\hline SS2 & 28000 & 23000 & 60000 \\
SS3 & 80000 & 75000 & 60000 \\
SS4 & 94000 & 80000 & 60000 \\
SS5 & 13000 & 8000 & 6000 \\
\hline
\end{tabular}

Figure 9 shows the comparison of the throughput of the node 5.The throughput is low because of its greater distance from the Base station. However the RED queue system tries to provide a reasonable throughput.

Table 3 shows the various values compared with the existing system. The throughput has increased and the jitter value is found for different users individually. There are factors like interference and attenuation which have an impact in the jitter value.

\section{DISCUSSION}

The results show that the throughput of the system, which consists a base station and four mobile users is calculated. The variations in the throughput of different users is due to the priority in their data and also due to the distance between them. The variations show that more often the users demand a high priority data or send a high priority dat. For the proposed algorithms Table 1 and $\mathbf{2}$ give the jitter values of corresponding users. The jitter has large variations in RED queue when compared with the
Priority queue. Table 3 gives the different throughput range for different users in both the proposed methods.

\section{CONCLUSION}

In this study, we proposed a scheduling scheme to support Quality of service in IEEE 802.16 standards. The two algorithms are almost able to give a throughput nearly equal to each other. But the Priority queueing gives better throughput for all the nodes compare to the Random Early Detection and EBA. Eventhough EBA gives a constant throughput it is suitable when the user's location is a a fixed one. But our proposed algorithms give high throughputs while the users are in mobile. The throughput of the Priority Queue system depends on the priority of the data that needs to be sending through the link. It ensures the delivery of the data, if the data is a high priority. Fluctuation prevails in every node as all the users send a mix of high and low priority data. In the previous work the jitter was not calculated for the different users. Our proposed algorithms give the various jitter values produced due to the mobility of the users.

\section{REFERENCES}

Alavi, H.S., M. Mojdeh and N. Yazdani, 2005. A quality of service architecture for IEEE 802.16 standards. Proceedings of the Asia-Pecific Conference on Communications, Oct. 5-5, IEEE Xplore Press, Perth, WA., pp: 249-253. DOI: 10.1109/APCC.2005.1554058

Barbu, O.E. and O. Fratu, 2011. An enabler of interoperability in heterogeneous wireless networks. Proceedings of the 2nd International Conference on Wireless Communication, Vehicular Technology, Information Theory and Aerospace and Electronic Systems Technology (Wireless VITAE), Feb. 28Mar. 3, IEEE Xplore Press, Chennai, pp: 1-5. DOI: 10.1109/WIRELESSVITAE.2011.5940885

Chen, S., A.M. Wyglinski, S. Pagadarai, R. Vuyyuru and O. Altintas, 2011. Feasibility analysis of vehicular dynamic spectrum access via queueing theory model. IEEE Commun. Mag., 49: 156-163. DOI: 10.1109/MCOM.2011.6069723

Chowdhury, P., A. Kundu, I.S. Misra and S.K. Sanyal, 2012. Analytical model for channel allocation scheme in macro/femto-cell based BWA networks. Proceedings of the International Conference on Recent Advances in Computing and Software Systems, Apr. 25-27, IEEE Xplore Press, Chennai, pp: 164-169. DOI: 10.1109/RACSS.2012.6212717 
Floyd, S. and V. Jacobson, 1993. Random early detection gateways for congestion avoidance. IEEE/ACM Trans. Network., 1: 397-413. DOI: 10.1109/90.251892

Jiang, C.H. and T.C. Tsai, 2006. Token bucket based CAC and packet scheduling for IEEE 802.16 broadband wireless access networks. Proceedings of the 3rd IEEE Computer Communication and Networking Conference, Jan. 8-10, IEEE Xplore Press, Taiwan, pp: 183-187. DOI: 10.1109/CCNC.2006.1593012

Jin, X. and G. Min, 2007. Performance modelling of priority queuing discipline under self-similar and poisson traffic. Proceedings of the 32nd IEEE Conference on Local Computer Networks, Oct. 1518, IEEE Xplore Press, Dublin, pp: 567-574. DOI: 10.1109/LCN.2007.124

Kim, J.G., I.C. Baek, H.K. Kang and J.S. You, 2011. Effective contention-based bandwidth request scheme for QoS enhancement in IEEE 802.16e. Proceedings of the 13th International Conference on Advanced Communication Technology (ICACT), Feb. 13-16, IEEE Xplore Press, Seoul, pp: 35-38.
Lai, D.N., T.C. Huang and H.Y. Chi, 2011. Efficient bandwidth allocation with QoS guarantee for IEEE 802.16 systems. Proceedings of the International Conference on Parallel Processing, Sept. 13-16, IEEE Xplore Press, Taipei City, pp: 115-119. DOI: 10.1109/ICPP.2011.81

Liu, Q., X. Wang and G.B. Giannakis, 2005. Cross-layer scheduler design with QoS support for wireless access networks. Proceedings of the 2nd International Conference on QoS in Heterogeneous Wired/Wireless Networks, Aug. 24-24, IEEE Xplore Press, Lake Vista, FL, pp: 8-15. DOI: 10.1109/QSHINE.2005.16

Taddia, C. and G. Mazzini, 2006. On the jitter performance of FIFO and priority queues mixture. Proceedings of the IEEE 17th International Symposium on Personal, Indoor and Mobile Radio Communications, Sept. 11-14, IEEE Xplore Press, Helsinki, pp: 1-5. DOI: 10.1109/PIMRC.2006.254045 